\title{
Fundus Image Segmentation using a Random Walks Approach
}

\author{
Jun XI \\ Department of Math \& Computer, Xinyu College, Xinyu, Jiangxi, China \\ 94671326@qq.com
}

\begin{abstract}
The fundus processing and analysis are with great significance in the medical and healthcare fields since medical signs that are detected from their observations such as hemorrhages, exudates, cotton wool spots, blood vessel abnormalities and pigmentation could be used for helping the doctors to make decisions on the treatment. This paper aims to introduce a random walks-based pre-processing approach for fundus image segmentation so as to address some difficulties which carrying out fundus image processing. Firstly, the pre-knowledge from fundus is extracted to deal with the tiny blood vessel. Secondly, the random walks-based approach is proposed to face the low contrast degree caused by the reflection effects from fundus. From the experiment results, it could be observed that the proposed model in this paper outperforms the manual operations and Hoover algorithm not only in the normal fundus images, but also in the images with large number of abnormalities. From 0 to 30 (specificity), random walks-based approach always outperforms the Hoover algorithm in sensitivity that because the proposed method uses different oriented Laplacian operator to deal with the low and high contrast vessels.
\end{abstract}

Keywords: Fundus Image, Random Walks, Segmentation, Divergence.

\section{Introduction}

The fundus of eyes is the interior surface of the eye opposite the lens that include retina, optic disc, macula, fovea, and posterior pole, which can be examined by an ophthalmoscopy or fundus photography [1]. The fundus processing and analysis are with great significance in the medical and healthcare fields. Medical signs that are detected from their observations such as hemorrhages, exudates, cotton wool spots, blood vessel abnormalities and pigmentation could be used for helping the doctors to make decisions on the treatment [2]. For the humans, the fundus image is the only part where the microcirculation can be observed directly. For instance, the diameter of the blood vessels around the optic disc are about $150 \mu \mathrm{m}$, and an ophthalmoscope allows observation of blood vessels as small as $10 \mu \mathrm{m}$ [3].

As the cutting-edge technologies and concepts used widely in our daily life, advanced decision-makings are achieved in recent years such as the Internet of Things (IoT) enabled manufacturing and logistics [4], Cloud-based healthcare and planning \& scheduling [5], as well as Big Data Analytics for services and supply chain management [6]. However, the computer vision field is lagged compared to the other research areas like manufacturing and aerospace [7, 8]. Take fundus image processing/analysis for example, there are several reasons for the lag. In the first place, there are lots of abnormalities for example cotton wool spots which makes higher difficulties to observe due to the lack of depth appreciation. Different tissues and parts are changing from shapes to sizes. There is no standards for differentiate the same parts. Secondly, cost of processing these images are extremely high so that most the decision-makings are based on manual operations. Since there are many causes like cataracts which will reduce image clarity, there is less magnification and image clarity than indirect ophthalmoscopy, especially retinal conditions such as macular degeneration and diabetic retinopathy. 
Currently, large number of studies have been carried out for promoting the fundus image processing from theoretical and practical perspectives. Zilly et al proposed a novel convolutional neural network (CNN) based approach for optic cup and disc segmentation from fundus images [9]. Through the experiments, the proposed algorithm outperforms existing methods on the public DRISHTI-GS data set. Sridhar et al introduced an efficient blood vessel segmentation approach for color fundus images [10]. Local-entropy-based thresholding and modified Gaussian-based matched filter segmentation techniques are used to assess the information of blood vessels. Deepashri and Santhosh gave a comprehensive review on the Glaucoma detection by image fusion from fundus color retinal images [11]. They discussed the different automated techniques developed for the glaucoma detection which is independent of image quality and invariant to noise to screen glaucoma.

Despite all the efforts placed on the research, there are still some research gaps to be fulfilled. This paper aims to introduce a random walks-based pre-processing approach for fundus image segmentation so as to address some difficulties which carrying out fundus image processing. Firstly, the pre-knowledge from fundus is extracted to deal with the tiny blood vessel. Secondly, the random walks-based approach is proposed to face the low contrast degree caused by the reflection effects from fundus. That may result in the nonaverage lightness of various parts or tissues. Then finally, a segmentation method is presented to pick up the blood vessels from the color fundus images. In order to illustrate this paper properly, the following sections are organized. Section 2 gives a brief literature review about related works. Section 3 introduces the analysis of blood vessels characteristics from fundus images. Section 4 presents pre-knowledge extraction approach. Section 5 describes the random walks-based segmentation model. Section 6 demonstrates experimental results and discussions. Section 7 gives a conclusion and future research directions.

\section{Literature Review}

Related work about the fundus image processing includes two aspects: theoretical and practical. From theoretical aspect, large number of studies focus on the models, algorithms, and different approaches. Udayakumar et al introduced a coupled sparse dictionary method for depth-based cup segmentation from single color fundus image [12]. This paper innovatively presents a framework for depth based optic cup boundary extraction from a single 2D color fundus photograph per eye, where multiple depth estimates from shading, color and texture gradients in the image are correlated with Optical Coherence Tomography (OCT) that is based on depth using a coupled sparse dictionary, trained on image-depth pairs. Out of the pathological features from fundus images, exudates and hemorrhages cause severe vision loss when they appear over the macula. Thus, the exudates have been extracted using Selective Binary and Gaussian filtering Regularized Level Set (SBGFRLS) by implementing it on blood vessels inpainted image [13]. The examination of the optic nerve head is difficult based on fundus image as glaucomatous patients tend to have larger cup-to-disc ratios. [14] proposed a novel algorithm for optic nerve head segmentation which uses template matching to find the region of interests. Because of the location of an area that considered as pathological blood vessels when in segmentation is very difficult, [15] uses K-means clustering to detect the optic disc area. This model successfully detects optic disc area quickly and segmented blood vessels more quickly compared with traditional approaches. The attention of human visual systems directs is to regions instead of points for feature matching. Being aware of these issues, [16] presented a new structure-based region detector, which identifies stable and distinctive regions, to find correspondences. This paper shows the experimental results on four datasets including temporal and partially overlapping image pairs that the approach is comparable or superior to SIFT-based 
methods in terms of efficiency, accuracy and speed with successfully registered $92.30 \%$ of 130 temporal image pairs and $91.42 \%$ of 70 different field of view image pairs.

For practical aspect, some real-life implementations have been studied and presented. Arun and Sasirekha presented a real-life application of fundus image to diagnose the diabetes [17]. A new algorithm is proposed in their work to detect the presence of hemorrhage with maximum efficiency and accuracy by partitioning the image into differentiated segments covering the entire retinal image. Yu et al demonstrated a new, fast, and robust methodology for fully automatic localization and segmentation of the optic disc in fundus images in practice [18]. Their experimental results show that location procedure has a high success rate of $99.52 \%$ in the cases averagely and the segmentation method improves the sensitivity and specificity to $99.92 \%$ and $96.49 \%$ respectively. Image segmentation helps to analyze images by simplifying the representation of image. Bauzet et al used a universal algorithm for fundus image segmentation based on multiscale blood vessel segmentation in retinal fundus images algorithm [19]. In the practical implementation, it was observed that the blood vessel can be identified using the multiscale blood vessel segmentation in retinal fundus images algorithm. It also found that the preprocessing of the captured fundus images is very essential, thus the results can be further enhanced by using selective and regional image smoothing functions according to the fundus images characteristics before applying the multiscale blood vessel segmentation in retinal fundus images algorithm.

From the literature review, it could be summarized that the fundus image processing especially the segmentation is still in the initial stage due to the complexity and various implementation cases. Further studies on the efficiency improvement and accuracy enhancement should be carried out so that the automatic detection mechanisms would be achieved. Thus, this paper introduces a random walks-based pre-processing approach for the fundus image segmentation so that further analysis like 3-D model reconstruction could be carried out.

\section{Proposed Approach}

\subsection{Analysis of Blood Vessels Characteristics in Fundus Images}

A typical fundus image includes retina, optic disc, macula, fovea, posterior pole, and blood vessels. The most important part is blood vessels which are usually tiny. As we known in the human body, there are two types of vessels: arteries and veins. Arteries are brighter from an image because they transport blood rich in oxygen to the organs; while the veins are darker afterwards transport the blood that is at a low oxygen level. However, in the fundus images, the blood vessels are always with low contrast. Additionally, due to the abnormities of some tissues in our eyes, the vessels are variable in terms of shape and brightness. That will cause extreme difficulties when carrying out segmentation.

From some observations, the characteristics of blood vessels from fundus images could be summarized as follows. Firstly, the blood vessels in our eyes are commonly extending to different directions to other parts like retina from optic disc. The distances among them are very close so that a network with crossed structure is formed. Secondly, the brightness of the blood vessels is darker than the background from fundus images that because most of the blood vessels are not obvious from the image compared with other tissues like optic disc and fovea. Thirdly, the shapes of blood vessels are long pipe chains which have partial linear structure. Compared with other components in the eyes, blood vessels are the only components which may have linear styles.

\subsection{A Pre-knowledge Extraction Approach}

Based on the characteristics of blood vessels from fundus images, a pre-knowledge extraction approach is presented. For a typical fundus image, there are major three parts 
related to this research. They are blood vessels, background, and abnormities. For the segmentation of blood vessels, it is difficult to implement if we are trying to remove the abnormities which are usually with high uncertainty. Thus, in this paper, based on the long pipe chain with linear characteristics, a pre-knowledge extraction approach could be established for working out the features. The sections of the blood vessels are similar with Gauss distribution. This approach is based on the divergence of vector from the image gradient to extract the center line of the blood vessels. That could be presented as a line trends so that the pre-knowledge could be provided.

Divergence, in vector calculus, is a vector operator that produces a signed scalar field giving the quantity of a vector field's source at each point which represents the volume density of the outward flux of a vector field from an infinitesimal volume around a given point $[25,26]$. Assume $M_{0} \in \Omega, V$ is an area which includes $M_{0}, V \subset \Omega . \Sigma$ presents the exterior of $V . n$ is the vector of $\Sigma$. The divergence of vector field $F$ in $M_{0}$ could be expressed as:

$$
\operatorname{div} F\left(M_{0}\right)=\lim _{V \rightarrow M_{0}} \frac{\iint_{\Sigma} F \llbracket n}{|V|} d S
$$

Where $|V|$ is the volume of $V . S(V)$ is the boundary of $V$, and the integral is a surface integral with $n$ being the outward unit normal to that surface. $d i v F$ is a function of $M_{0}$. From this definition it also becomes explicitly visible that $d i v F$ can be seen as the source density of the flux of $F$. For the points which meet $\operatorname{div} F>0$, they are sources. And for the points satisfying $\operatorname{div} F<0$, they are convergence. If $\operatorname{div} F=0$, they are non-source.

Compared with the other parts in fundus images, the blood vessels are usually darker. They could be regarded as sources. Thus, it is possible to establish the gradient vector divergence based on the blood vessel characteristics to achieve the segmentation of the fundus images.

For a fundus image $f\left(x^{\prime}, y^{\prime}\right)$, Gauss filter $G\left(\eta^{2}\right)$ is used for the image smoothening.

$$
g\left(x, y ; \eta^{2}\right)=f\left(x^{\prime}, y^{\prime}\right) \oplus G\left(\eta^{2}\right)
$$

Where $\oplus$ is the convolution operation. The gradient vector $F$ of the $g\left(x, y ; \eta^{2}\right)$ is

$$
F(x, y)=\frac{\partial g\left(x^{\prime}, y^{\prime} ; \eta^{2}\right)}{\partial x} i \oplus \frac{\partial g\left(x^{\prime}, y^{\prime} ; \eta^{2}\right)}{\partial y} j
$$

Then, rotate the $F(x, y)$ with $\theta$ using the rotation matrix $R_{\theta}$, the rotated gradient $F_{\theta}$ could be obtained: 


$$
\begin{aligned}
F_{\theta}(x, y)= & R_{\theta} F(x, y)=\left[\frac{\partial g\left(x^{\prime}, y^{\prime} ; \eta^{2}\right)}{\partial x} \tan \theta-\frac{\partial g\left(x^{\prime}, y^{\prime} ; \eta^{2}\right)}{\partial y} c \tan \theta\right] i \oplus \\
& {\left[\frac{\partial g\left(x^{\prime}, y^{\prime} ; \eta^{2}\right)}{\partial x} \cos \theta+\frac{\partial g\left(x^{\prime}, y^{\prime} ; \eta^{2}\right)}{\partial y} \sin \theta\right] j }
\end{aligned}
$$

Thus, $\operatorname{div} F\left(\theta, \eta^{2}\right)$ could be calculated according to definition (1):

(5)

$$
\begin{aligned}
\operatorname{div} F\left(\theta, \eta^{2}\right)= & \left(\frac{\partial^{2} g\left(x^{\prime}, y^{\prime} ; \eta^{2}\right)}{\partial x^{\prime 2}} \tan \theta+\frac{\partial^{2} g\left(x^{\prime}, y^{\prime} ; \eta^{2}\right)}{\partial y^{\prime 2}} \cos \theta\right) \oplus \\
& \left(\frac{\partial^{2} g\left(x^{\prime}, y^{\prime} ; \eta^{2}\right)}{\partial x^{\prime 2}} \sin \theta+\frac{\partial^{2} g\left(x^{\prime}, y^{\prime} ; \eta^{2}\right)}{\partial y^{\prime 2}} c \tan \theta\right)
\end{aligned}
$$

$\operatorname{div} F\left(\theta, \eta^{2}\right)$ is also termed as Oriented Laplacian operator. Using the same approach, the normalized gradient vector field $F=F /\lfloor F\rfloor$ could be obtained and with the divergence is $\operatorname{div} F\left(\theta, \eta^{2}\right)$.

The contrast of the blood vessels from a fundus image may be different. Typically, the main blood vessels have the stronger contrast; while the end of the vessels or the vessels in an abnormal area have weaker contrast. In order to extract the center line of the blood vessels, two categories are used for mining the two types of vessels.

\subsection{High Contrast Vessels}

For the high contrast blood vessels, smaller smoothing parameter is selected $\eta_{H}=0.0+0.20 \mathrm{~m}, m \in[0,40]$. The rotation $\theta_{n}=0+n \pi / 360, n \in[0,180]$. The normalized gradient vector field divergence $\operatorname{div} F\left(\theta_{n}, \eta_{m}^{2}\right)$ could be obtained and it must meet:

$$
\operatorname{div} F\left(\theta, \eta^{2}\right)>F=F /\lfloor F\rfloor
$$

For the blood vessel center line $h_{c}\left(x^{\prime}, y^{\prime} ; \theta_{n}, \eta_{m}^{2}\right)$, due to the abnormity, there are some noises. In order to get rid of these noises, the length filter approach could be used.

\subsection{Low Contrast Vessels}

In order to extract the vessels center line from low contrast vessels, bigger smoothing parameter is used $\eta_{m}=3.0+0.20 \mathrm{~m}, m \in[0,50]$. The rotation $\theta=0^{\circ}$ meets the condition:

$$
\max _{\eta_{m}}\left\{\frac{\operatorname{div} F\left(0, \eta_{m}^{2}\right)}{f \oplus G\left(\sigma^{2}\right) / m \times n}\right\}
$$

For the blood vessel center line $l_{\text {center }}\left(x^{\prime}, y^{\prime} ; \theta_{n}, \eta_{m}^{2}\right)$, the filter approach is also used for reducing the noises. 


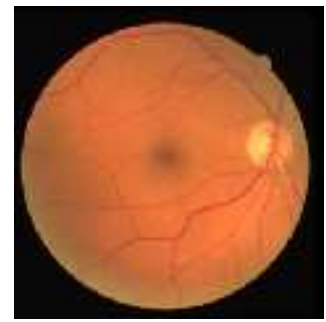

(a)

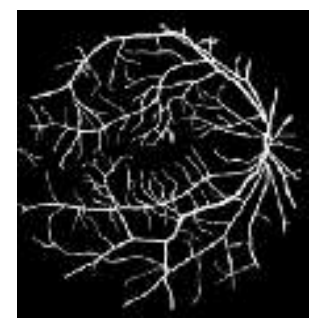

(c)

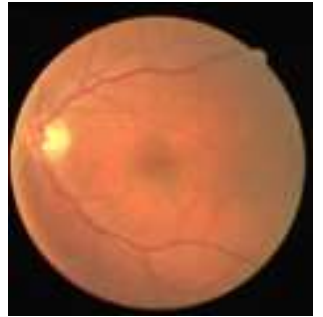

(b)

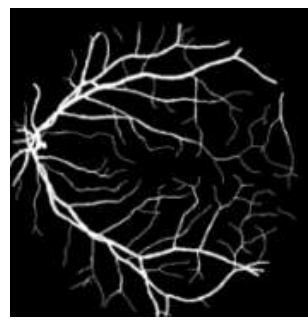

(d)

Figure 1. Extraction of Vessel Center Lines

In order to evaluate the extraction approach, Figure 1 presents the experimental results for both high and low contrast vessels center line extraction. Figure 1 (a) and (b) are two fundus images from the STARE fundus image database. Figure 1 (c) is the high-contrast center lines extraction result and Figure 1 (d) is the low-contrast center lines extraction results. It could be observed that the proposed approach is able to figure out the center lines effectively. Additionally, the abnormality will not affect the experimental result in this method. Thus, the proposed approach could be feasible for the pre-knowledge extraction.

\section{Random Walks-based Segmentation Model}

Random walks theory has been widely used in economic game and computer networks with significant success. This paper uses the random walks theory twining the preknowledge extraction approach to carry out the blood vessels segmentation. To make full use of the center lines, the maximum probability of the pixel arriving at the center lines is selected via the random walk model, aiming to segment the image pixels into different sets.

\subsection{Mapping Approach}

The mapping approach is very important when using the pre-knowledge random walks model into image segmentation. Assume an image $I_{\text {image }}(x, y)$ which should be mapped into $\operatorname{Graph}=(V, E)$ which has a limited vertices set $v_{i} \in V$ and edges $e_{j} \in E \subseteq V \times V$. Edge $e$ connects the vertices $v_{i}$ and $v_{j}$, labelling as $e_{i, j}$. Each edge in the weighted

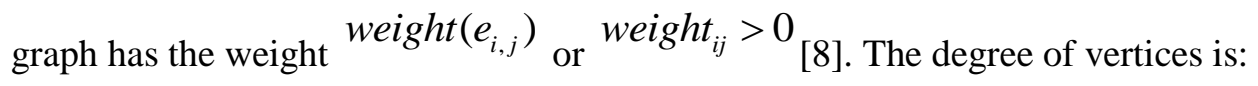

$$
d_{i}^{\text {deg ree }}=\sum \text { weight }\left(e_{i, j}\right), \forall e_{i, j} \in E
$$


Mapping the fundus image $I_{\text {image }}(x, y)$ into $\operatorname{Graph}=(V, E)$ follows the steps: each pixel $i$ in the image $I_{\text {image }}$ is mapped into a vertex $v_{i}$ in the Graph. Based on the Gaussian weight function, the weight $w_{i j}$ of the edge $e_{i, j}$ could reflect the brightness of the pixel.

$$
\text { weight }\left(e_{i, j}\right)=\exp \left[-\alpha \frac{\left(b r_{i}-b r_{j}\right)^{2}}{\beta}\right] \oplus \lambda
$$

Where $\lambda=10^{-4}$ is a small constant which ensures weight $\left(e_{i j}\right)>0 . b r_{i}$ indicates the brightness of pixel $i$. The normalized parameter $\beta=\max \left(b r_{i}-b r_{j}\right), \forall i, j$. The adjustment parameter is $\alpha=0.3 \times 10^{3}$.

\subsection{Segmentation Model}

Based on the mapping approach, the first arrival probability of the pixels to the center lines could be worked out by the random walks theory. The probability could be converted into Dirichlet problem which is the problem of finding a function which solves a specified partial differential equation (PDE) in the interior of a given region that takes prescribed values on the boundary of the region. The boundary condition $\Gamma$ for $I_{\text {image }}(x, y)$ should meet the Laplace equation:

$$
\begin{aligned}
& \Gamma^{2} u \equiv \frac{\partial^{2} u}{\partial x^{2}}+\frac{\partial^{2} u}{\partial y^{2}}=0 \\
& \left.u(x, y)\right|_{\Gamma}=\left\{\begin{array}{c}
1,(x, y)=u \\
0, \text { Others }
\end{array}\right.
\end{aligned}
$$

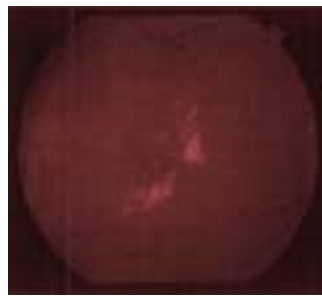

(a)

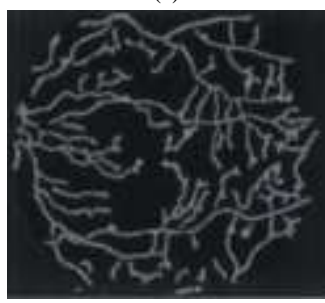

(c)

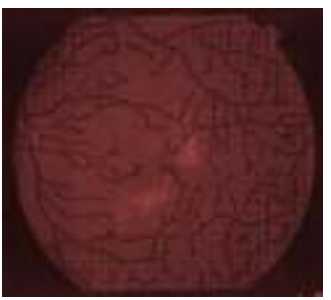

(b)

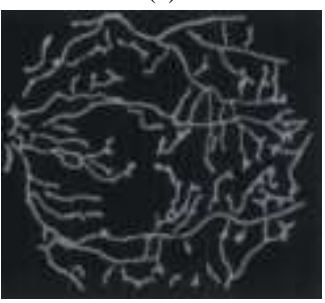

(d)

Figure 2. Segmentation Evaluations

In order to test the segmentation approach, a simple evaluation is carried out. Figure 2 shows the experimental results. Figure 2 (a) is the original fundus image. (b) is the vessel center lines overlaid on the image which is obtained from the pre-knowledge approach. 
(c) presents the first arrival probability results using the proposed method in this section. (d) is the result of the vessel segmentation. From this simple experiment, it could be found that the proposed method not only extracts the high contrast vessels, but also works well on the tiny vessels from segmenting the vessels from the background. The noises are reduced with great proportion so that they are not able to influence the segmentation results.

\section{Experiment Results and Discussions}

In this section, two types of experiments are carried out. First of all, two fundus images are randomly selected from DRIVE database for examining the proposed method in this paper. Figure 3 shows the experimental results which include four categories.

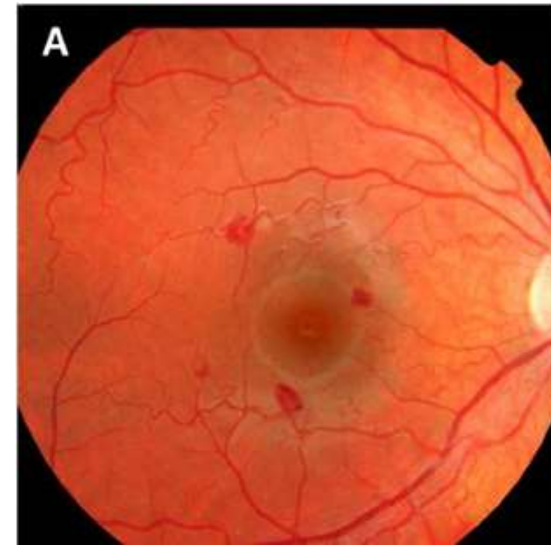

(a)

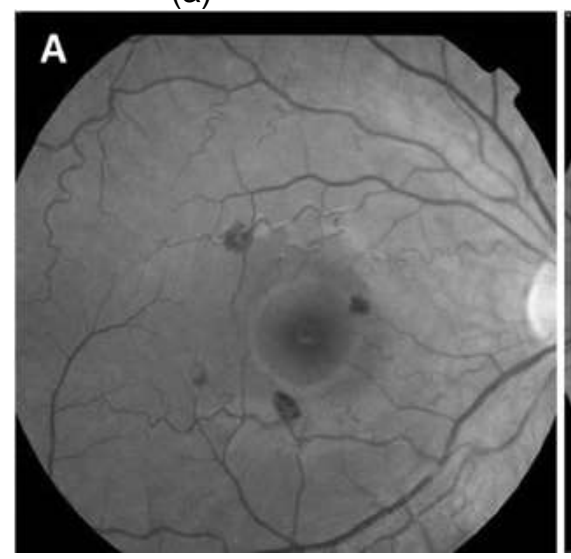

(c)

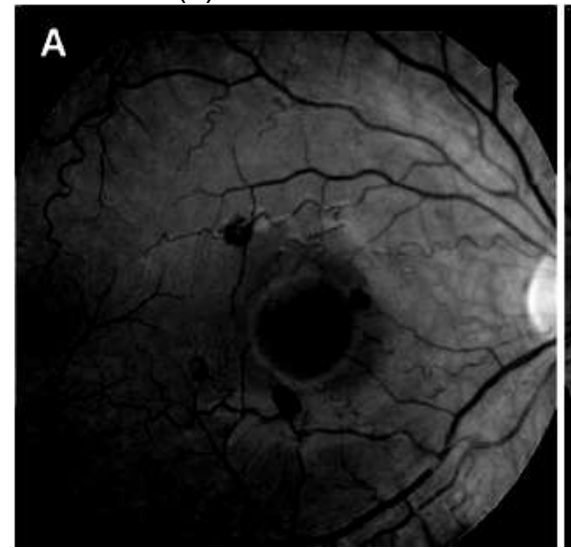

(e)

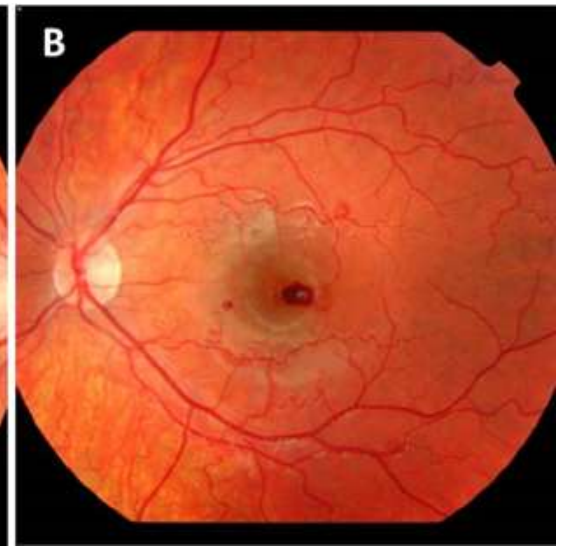

(b)

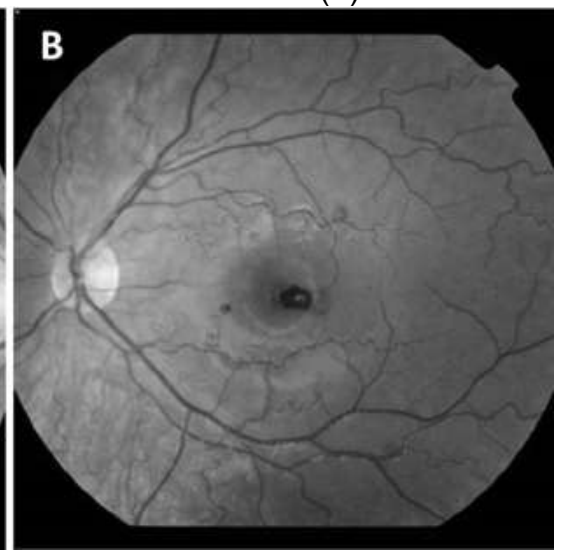

(d)

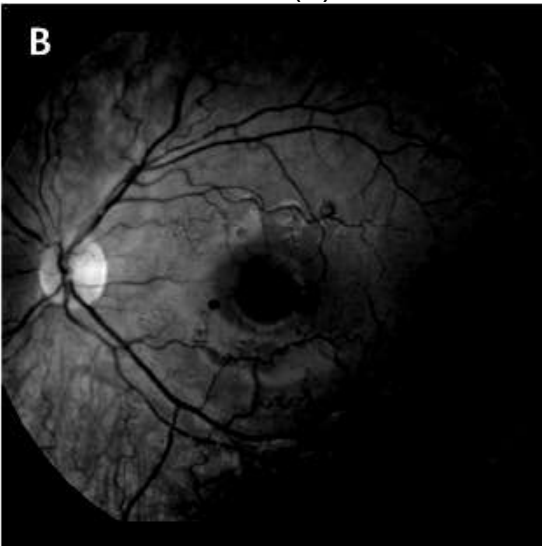

(f) 


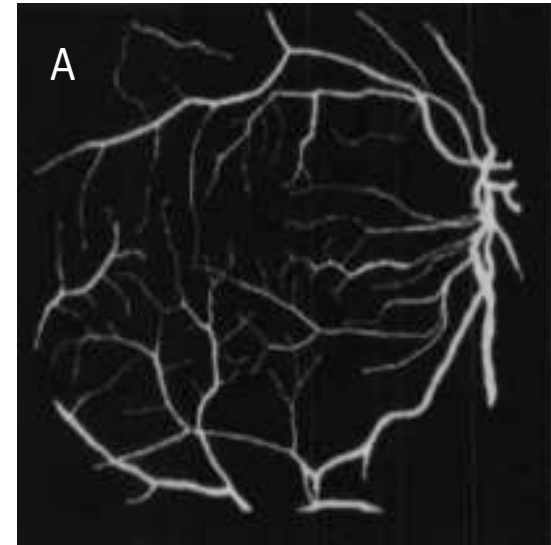

(g)

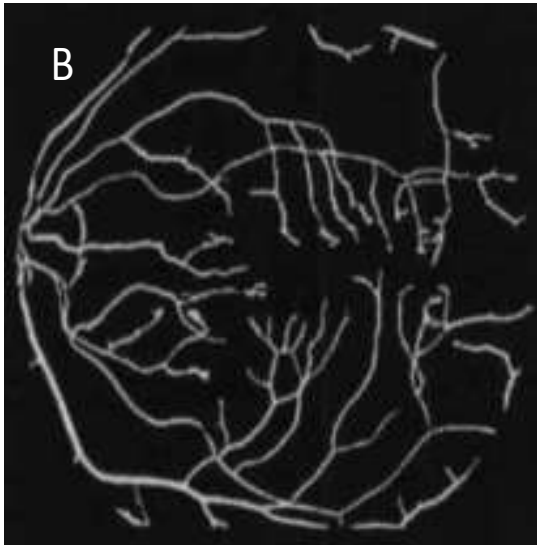

(h)

Figure 3. Segmentation Results

Figure 3 (a) and (b) are the original colored fundus images from DRIVE database. They are right and left eye with random picking up from the database. (c) and (d) is the results from implementing pre-processing. It could be seen that after the pre-processing, the figures are much smoother and some noises are removed. The edges of each components like vessels and optic disc could be clearly identified. (e) and (f) are the results from removing the background. From these two images, the vessels are identified by figuring out the center lines and their characteristics could be marked. (g) and (h) are the segmented results which are efficient and effective for labelling the blood vessels. The high and low contrast vessels are clearly worked out so that the proposed approach is feasible for practical implementations.

Second experiment is carried out to compare the proposed approach with Hoover algorithm and manual operations which are widely used in the image segmentation. The experiment is based on the public Hoover STARE fundus images database for selecting the samples. The experimental parameters are $\phi_{1}=0.4+0.05(k-1), k=4$, and $\phi_{2}=1$. This experiment is based on Matlab 2008 platform with $1.60 \mathrm{GHz}$ iCore CPU, 4GB RAM. The average time for processing an image is within two minutes. Thus, if using the applicable programming language like $\mathrm{C}$ and $\mathrm{C}++$, the calculation speed will be much faster. Figure 4 shows the experiment results.

Figure 4 presents the compared results with Hoover manual operations and Hoover method. The first row shows the original fundus images that are selected from the database. The second row is based on the manual operations processed by the ophthalmologist who has rich experience in diagnosing the disease of eyes. The third row is the results from Hoover algorithm which is used for the segmentation. The fourth row is the results from the approaches proposed in this paper. For the four different fundus images, the first one is a common eye image. The second one has a low contrast vessels. 


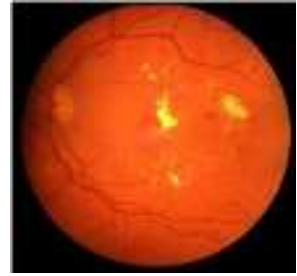

(a)

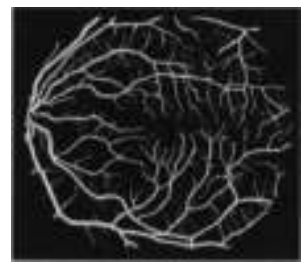

(a1)

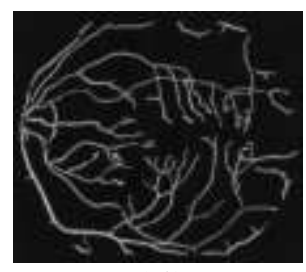

(a2)

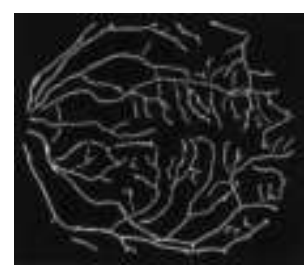

(a3)

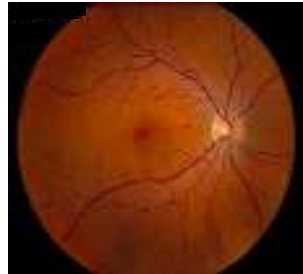

(b)

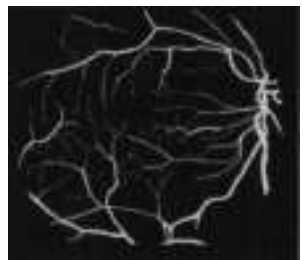

(b1)

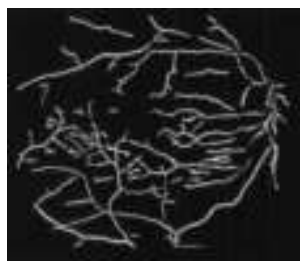

(b2)

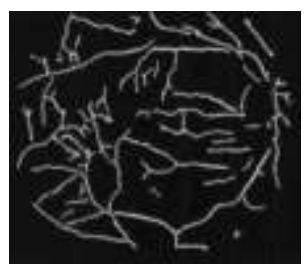

(b3)

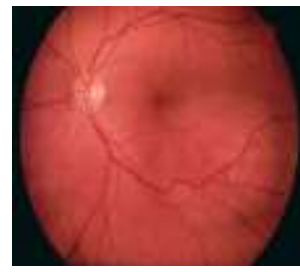

(c)

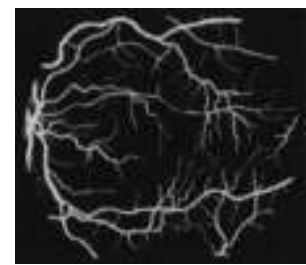

(c1)

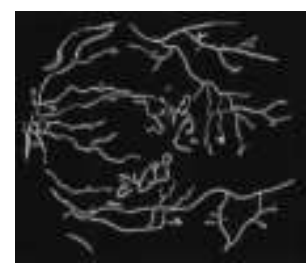

(c2)

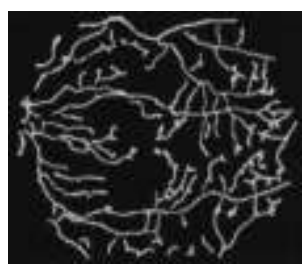

(c3)

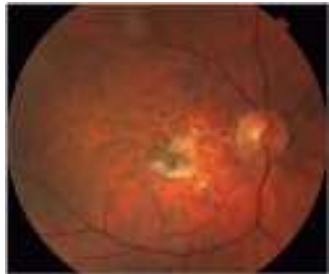

(d)

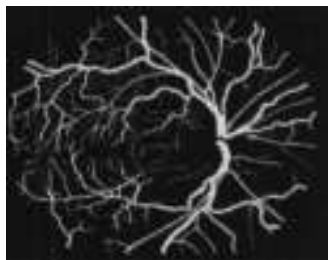

(d1)

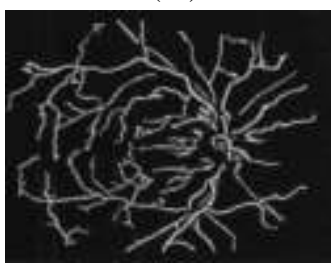

(d2)

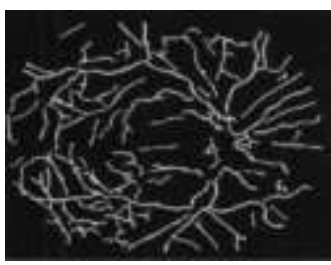

(d3)

Figure 4. Comparison Results

The third and the fourth images have large number of abnormalities which will affect the segmentation. It could be observed from Figure 4, the proposed model in this paper outperforms the other two approaches not only in the normal fundus images, but also in the images with large number of abnormalities. The outperformance comes from the segmented blood vessels which are more completed and some tiny vessels are figured out. Such tiny vessels are hardly observed from the segmented results by Hoover algorithm. Additionally, the connection of the segmented vessels are much better than the results from Hoover algorithm $(\mathrm{H})$. Some non-connected vessels are combined in the last row images. 


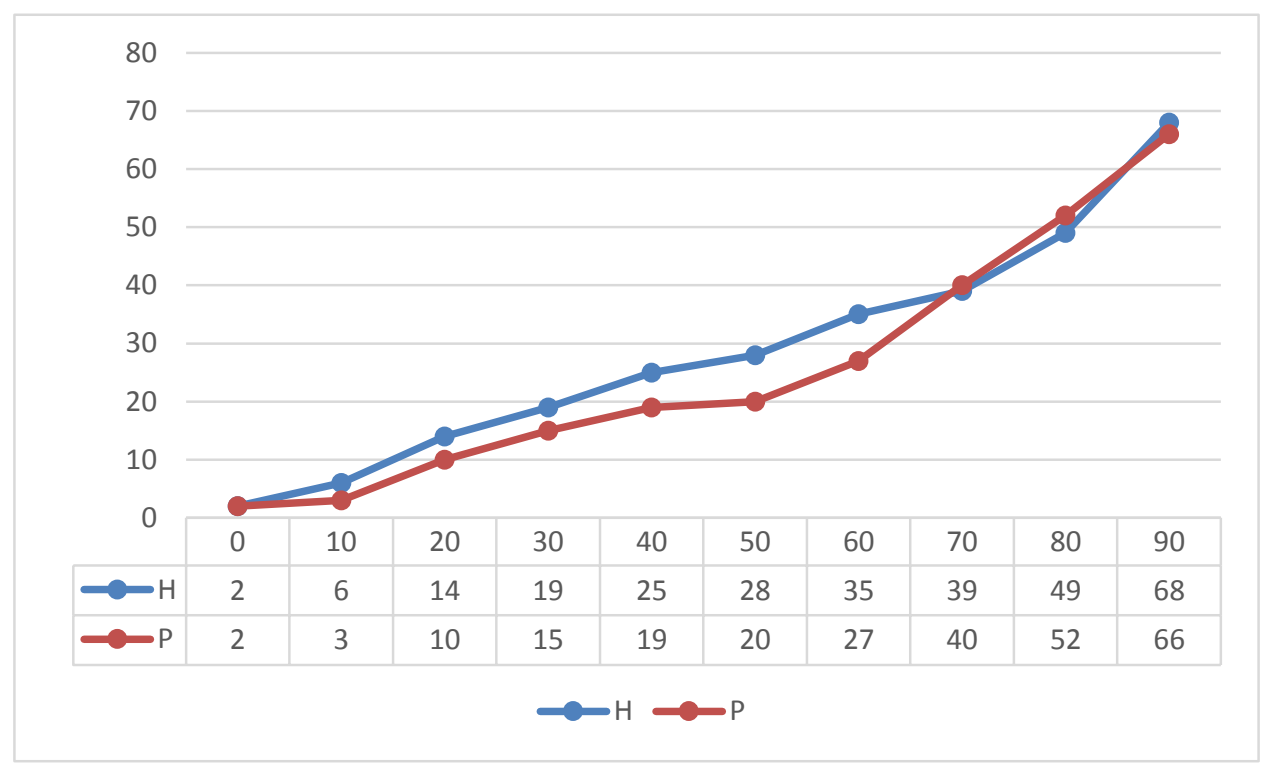

Figure 5. ROC Curve Comparison

In order to further evaluate the performance, receiver operating characteristics curve (ROC) is used for quantitatively analyzing the segmentation precisions. X-axis is the specificity $\%$ and $\mathrm{Y}$-axis is the sensitivity $\%$. From Figure 5, it could be observed that with the same specificity, the proposed model in this paper has better sensitivity than Hoover algorithm. That means with the same error ratio, our approach is able to segment more blood vessels. As the increasing of specificity, the sensitivity of proposed method and Hoover algorithm are almost the same.

For detailed analysis, from 0 to 30 (specificity), random walks-based approach always outperforms the Hoover algorithm in sensitivity that because the proposed method uses different oriented Laplacian operator to deal with the low and high contrast vessels. From 30 to 42 , Hoover algorithms slightly outperforms the proposed approach. And after that, their performances are almost the same. The proposed method is able to get better performance under abnormal fundus images so that the practical implementation could be achieved in eye diseases diagnose.

\section{Conclusion and Future work}

This paper introduced a random walks-based segmentation approach for fundus images processing. The characteristics of fundus images, take blood vessels for example, were discussed so as to figure out the vessels' presentations. A pre-knowledge extraction method was proposed to address the high and low contrast of the blood vessels using divergence mechanism. After that, a random walks-based segmentation model was presented by calculating the first arrival probability to categorize the pixel sets which are close to the center lines.

Future research directions may follow the two aspects. Firstly, the robustness of vessel segmentation should be enhanced. Thus, the discrimination feature like phase congruency of the vector field will be constructed to achieve this purpose. Secondly, automatic segmentation models using machine learning algorithms could be established. Based on the extreme learning machine, a set of discriminative feature vectors could be built up so that local features, morphological characteristics, and vessels shapes could be precisely presented. 


\section{References}

[1] K. Udayakumar, P. P. Bharati, S. Verma, and M. K. Nath, "Automatic Estimation of Vision Degradation from Color Fundus Image," International Journal of Image, Graphics and Signal Processing (IJIGSP), vol. 7, pp. 26, (2015).

[2] A. A. Salam, M. U. Akram, K. Wazir, S. M. Anwar, and M. Majid, "Autonomous Glaucoma detection from fundus image using cup to disc ratio and hybrid features," Proceeding in 2015 IEEE International Symposium on Signal Processing and Information Technology (ISSPIT), pp. 370-374, (2015).

[3] M. Basha and B. Jinaga, "A New Approach Based on Order Reduction Using Sub Image Formation in Minimizing the Computation Time for Image Compression," International Journal of Signal Processing, Image Processing and Pattern Recognition, vol. 8, pp. 337-346, (2015).

[4] R. Y. Zhong, Q. Y. Dai, T. Qu, G. J. Hu, and G. Q. Huang, "RFID-enabled Real-time Manufacturing Execution System for Mass-customization Production," Robotics and Computer-Integrated Manufacturing, vol. 29, pp. 283-292, (2013).

[5] R. Y. Zhong, S. T. Newman, G. Q. Huang, and S. Lan, "Big Data for supply chain management in the service and manufacturing sectors: Challenges, opportunities, and future perspectives," Computers \& Industrial Engineering, (2016).

[6] R. Y. Zhong, S. Lan, C. Xu, Q. Dai, and G. Q. Huang, "Visualization of RFID-enabled shopfloor logistics Big Data in Cloud Manufacturing," The International Journal of Advanced Manufacturing Technology, vol. 84, pp. 5-16, (2016).

[7] R. Y. Zhong, G. Q. Huang, S. L. Lan, Q. Y. Dai, C. Xu, and T. Zhang, "A Big Data Approach for Logistics Trajectory Discovery from RFID-enabled Production Data," International Journal of Production Economics, vol. 165, pp. 260-272, (2015).

[8] R. Y. Zhong, Z. Li, A. L. Y. Pang, Y. Pan, T. Qu, and G. Q. Huang, "RFID-enabled Real-time Advanced Planning and Scheduling Shell for Production Decision-making," International Journal of Computer Integrated Manufacturing. , vol. 26, pp. 649-662, (2013).

[9] J. G. Zilly, J. M. Buhmann, and D. Mahapatra, "Boosting convolutional filters with entropy sampling for optic cup and disc image segmentation from fundus images," in Machine Learning in Medical Imaging, ed: Springer, pp. 136-143, (2015).

[10] S. Sridhar, S. Rao, N. Hemanth, and M. K. Nath, "An Efficient Blood Vessel Segmentation from Color Fundus Image," International Journal of Computer Applications, vol. 119, (2015).

[11] K. M. Deepashri and K. Santhosh, "Glaucoma Detection by Image Fusion from Fundus Color Retinal Images: A Review," International Journal of Control Theory and Applications, vol. 8, pp. 1147-1152, (2015).

[12] K. Udayakumar, P. P. Bharati, S. Verma, and M. K. Nath, "Automatic Estimation of Vision Degradation from Color Fundus Image," International Journal of Image, Graphics and Signal Processing (IJIGSP), vol. 7, pp. 26, (2015).

[13] A. K. Whardana and N. Suciati, "A Simple Method for Optic Disk Segmentation from Retinal Fundus Image," International Journal of Image, Graphics and Signal Processing, vol. 6, pp. 36-42, (2014).

[14] Z. Ghassabi, J. Shanbehzadeh, and A. Mohammadzadeh, "A structure-based region detector for highresolution retinal fundus image registration," Biomedical Signal Processing and Control, vol. 23, pp. 5261, (2016)

[15] G. Arun and N. Sasirekha, "Detection of retinal hemorrhage in color fundus image using splat feature segmentation," Proceeding in Innovations in Information, Embedded and Communication Systems (ICIIECS), 2015 International Conference on, pp. 1-5, (2015).

[16] R. Y. Zhong, Q. Y. Dai, K. Zhou, and X. B. Dai, "Design and Implementation of DMES Based on RFID," Proceeding in 2nd International Conference on Anti-counterfeiting, Security and Identification, Guiyang, 20-23 Aug. pp. 475-477, (2008).

[17] R. Lambiotte, V. Salnikov, and M. Rosvall, "Effect of memory on the dynamics of random walks on networks," Journal of Complex Networks, vol. 3, pp. 177-188, (2015).

[18] R. Y. Zhong, G. Q. Huang, S. Lan, Q. Dai, T. Zhang, and C. Xu, "A two-level advanced production planning and scheduling model for RFID-enabled ubiquitous manufacturing," Advanced Engineering Informatics, vol. 29, pp. 799-812, (2015).

[19] C. Bauzet, G. Vallet, and P. Wittbold, "The Dirichlet problem for a conservation law with a multiplicative stochastic perturbation," Journal of Functional Analysis, vol. 266, pp. 2503-2545, (2014). 


\section{Author}

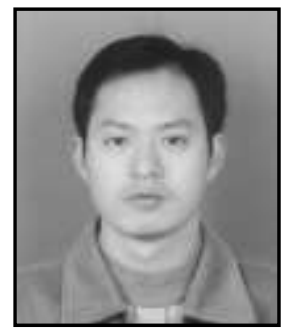

Jun Xi is current a lecturer from Department of Math \& Computer, Xinyu College. He graduated from East China Normal University with master degree of software engineering. His research interests include Image Processing, Computer Network and Security, as well as Database Technologies. He has published over 10 papers in international journals and conferences. 
International Journal of Signal Processing, Image Processing and Pattern Recognition Vol. 10, No. 8 (2017) 